\title{
Factores Asociados a Lesiones Músculo-Esqueléticas por Carga en Trabajadores Hospitalarios de la Ciudad de Torreón Coahuila México
}

\author{
MUSCULOSKELETAL LESIONS WHILE LIFIING-RELATED FACTORS IN WORKERS AT 3 HOSPITALS IN \\ TORREON, COAHUILA, MEXICO
}

Mario A. Rivera Guillén ${ }^{13}$, Maria F. Sanmiguel Salazar'1, Luis B. Serrano Gallardo', Martha P. Nava Hernández ${ }^{4}$, Javier Moran Martínez Luis C. Figuerola Chaparro², Eduardo E. Mendoza Mireles ${ }^{5,1}$, José J. García Salcedo'

1. Departamento de Bioquímica, Farmacología y Toxicología Centro de Investigación Biomédica Facultad de Medicina de Torreón Universidad Autónoma de Coahuila.

2. Estudiante de la Carrera Médico Cirujano y Becario de Investigación del Departamento de Bioquímica del Centro de Investigación Biomédica Facultad de Medicina de Torreón Universidad Autónoma de Coahuila.

3. Catedrático de Licenciatura en Fisioterapia. Universidad del Valle de México Campus Torreón.

4. Investigadores del Centro de Investigación Biomédica, Facultad de Medicina de Torreón Universidad Autónoma de Coahuila.

5. Departamento de cirugía y ortopedia del hospital Sørlandet sykehus en Flekkefjord, Noruega.

\section{RESUMEN}

Objetivo. Identificar factores asociados en la aparición de lesiones músculo-esqueléticas por carga en trabajadores hospitalarios. Métodos. Se aplicó el cuestionario estandarizado nórdico de sintomatologías músculo-esqueléticas con corte transversal que evaluó la frecuencia y factores asociados para las lesiones músculo-esqueléticas en 48 trabajadores. Resultados. La media de edad en hombres fue de $29,1+6,7$ y en mujeres $26,5+6,9$. El turno matutino tiene mayor actividad laboral. El 43,75\% eran paramédicos y el 22,92\% enfermeras. La espalda fue la región más afectada en los camilleros y paramédicos; mientras que enfermería reportó más dolor en pies y piernas. Se presentaron correlaciones significativas entre la antigüedad y los días que se presenta el dolor en la región anatómica. El 81,9\% no usa equipo de protección contra lesiones en espalda. El 91,67\% refirió capacitación previa para desarrollar actividades de cargas dentro del hospital. Conclusiones. Consideramos la importancia de la higiene ocupacional en las actividades hospitalarias y la capacitación del uso del equipo de protección personal en las actividades laborales que se realizan, la falta de uso puede ser uno de los factores determinantes para el incremento en los días con dolor en alguna región anatómica cuando incrementan los años laborables, principalmente lo que corresponde a espalda alta y baja.

(Rivera M, Sanmiguel M, Serrano L, Nava M, Moran J, Figuerola L, Mendoza E, García J, 2015. Factores Asociados a Lesiones MúsculoEsqueléticas por Carga en Trabajadores Hospitalarios de la Ciudad de Torreón Coahuila México. Cienc Trab. May-Ago; 17 [53]: 144-149).

Palabras clave: LESIÓN, SINTOMATOLOGÍA, MÚSCULO-ESQUELÉTICO, DOLOR.

\section{ABSTRACT}

Objective: To identify musculoskeletal lesions while lifting-related factors in hospital workers and lifting. Methods: A transversal study was carried out evaluating the prevalence and associated factors to musculoskeletal lesions in 48 workers, using the Nordic Standardized Questionnaire of musculoskeletal symptoms. Results: The mean age was $29.1+6.7$ for males and 26.5+6.9 for females. The morning shift had greater activity, Paramedics accounted for the $43.75 \%$ and nurses for the $22.92 \%$. Back pain was the most reported symptom among paramedics and auxiliary personnel; nurses reported feet and legs pain. Significant correlations were observed between seniority and the days with pain in the different anatomical regions. $81.9 \%$ of the workers do not use safety equipment against back injuries, and 91.67\% stated receiving previous training course on lifting activities within the hospital. Conclusions: We evaluated the importance of occupational hygiene within the hospital activities, as well as training of personal safety equipment. The lack of such may be a determining factor that increases days in pain in specific anatomic regions as the working years go by, particularly those pertaining to both upper and lower back.

Keywords: LESIONS, SYMPTOMATOLOGY, MUSCULOSKELETAL, PAIN.
Correspondencia / Correspondence:

Dr. Mario Alberto Rivera Guillén

Centro de Investigación Biomédica, Facultad de Medicina de Torreón,

Dpto. Bioquímica, Farmacología y Toxicología,

Universidad Autónoma de Coahuila.

Gregorio A. García 198 Sur Col. Centro Torreón Coahuila México. CP 27000

e-mail: riveramario_98@yahoo.com

Tel. 52- 8717176404 Ext. 25

Recibido: 13 de Abril de 2015 / Aceptado: 02 de Junio 2015

\section{INTRODUCCIÓN}

La carga de trabajo se define como el conjunto de requerimientos físicos y mentales a los que se somete un trabajador durante su jornada laboral, considerando la manipulación de cargas, las cuales pueden producir dolores dorso-lumbares y agotamiento muscular. ${ }^{1}$ Los riesgos de lesiones músculo-esqueléticas se presentan con frecuencia entre el personal de salud debido a que realizan una fuerza excesiva en determinadas tareas. ${ }^{2,3}$ 
Otros factores relacionados son la mala organización, sobrecarga del trabajo y factores ambientales, que supone estrés en determinadas regiones anatómicas. ${ }^{4}$

Algunas instituciones hospitalarias cuentan con condiciones ergonómicamente inadecuadas, lo cual produce enfermedades musculoesqueléticas en los trabajadores. ${ }^{4,5}$ Existe una necesidad de incorporar criterios ergonómicos en sus actividades, para prevenir estas enfermedades.

Las repercusiones ocasionadas por estas lesiones modifican en el trabajador su calidad de vida, asistencia, productividad, gastos en cuidados de su salud y cambios en las perspectivas y actitudes psicosociales individuales, familiares y sociales. $^{6}$

En Europa y Norteamérica se ha reportado que el dolor de espalda afecta más al personal de enfermería que a cualquier otra profesión, incluso las de la salud., ${ }^{2,7}$

En España la causa más frecuente de incapacidades laborales es la patología degenerativa de columna $(60,7 \%)$ y región lumbar (42,2\%). En Estados Unidos e Inglaterra se ha reportado la lumbalgia como la mayor causa de ausentismo en el personal de salud, $14 \%$ y $26,0 \%$ respectivamente. $^{8}$

En Argentina se evaluaron 74 trabajadores, el 59,5\% reportó problemas músculo-esqueléticos. El grupo más afectado fueron las mujeres de entre 25 a 33 años. ${ }^{9}$

En México se evaluaron las lesiones y ausentismo entre trabajadores de un hospital y las condiciones ergonómicas de trabajo, aplicando el cuestionario Nórdico de Síntomas osteomusculares. Las lesiones en columna lumbar y cervical fueron las más frecuentes $(59,68 \%){ }^{10,11}$ El objeto de nuestra investigación fue identificar factores asociados con la aparición de lesiones músculo-esqueléticas en trabajadores hospitalarios y evaluar la frecuencia del dolor en diferentes regiones anatómicas derivado de las actividades laborales de tres hospitales de la ciudad de Torreón Coahuila México.

Tabla 1.

Características de la población participante del estudio.

$\begin{array}{lccc}\text { Sexo } & \text { Hombre } & \% & n \\ & \text { Mujer } & 60,42 & (29) \\ \text { Edad } & \text { Sexo } & \text { Media } & (19) \\ & \text { Hombre } & 29,1+6,7 & (19-39) \\ & \text { Mujer } & 26,5+6,9 & (21-45) \\ \text { Peso } & \text { Hombre } & 80,8+7,3 & (69-98) \\ & \text { Mujer } & 56,4+25,9 & (54-79) \\ \text { Talla } & \text { Hombre } & 175,5+4,18 & (165-182) \\ & \text { Mujer } & 165,0+5,63 & (154-174) \\ \text { Grupos de edad } & \text { Rango de años } & \% & n \\ & 19-25 & 47,91 & (23) \\ & 26-35 & 29,17 & (14) \\ \text { Horario en que labora } & 36-45 & 22,91 & (11) \\ & & & \\ & \text { Matutino } & 39,58 & (19) \\ \text { Actividad dentro del hospital } & \text { Vespertino } & 29,17 & (14) \\ & \text { Nocturno } & 31,25 & (15) \\ & & & \\ & \text { Enfermeria } & 22,92 & (11) \\ \text { Antigüedad dentro de la institución } & \text { Chofer Ambulancia } & 16,67( & (8) \\ & \text { Rango en años } & & \\ & 1-5 & 56,25 & (27) \\ & 6-10 & 34,42 & (17) \\ & +10 & 8,33( & 4) \\ & & & \end{array}$

\section{MATERIAL Y MÉTODOS}

Estudio descriptivo de corte transversal con el objetivo de identificar los principales factores asociados en las lesiones músculoesqueléticas en trabajadores de tres hospitales públicos de la ciudad de Torreón Coahuila, México. Basados en el cuestionario estandarizado nórdico de síntomas osteomusculares ${ }^{11}$, se construyó un instrumento de recolección de datos sobre apreciación y sintomas de lesiones y dolor. Un total de 48 trabajadores aceptaron bajo carta de consentimiento informado participar en nuestro estudio, considerando como criterio de inclusión a quienes refirieron no haber presentado lesiones músculo-esqueléticas previas por alguna lesión de origen congénito o traumático. Para minimizar factores de confusión o sesgo, el cuestionario se aplicó en los diferentes departamentos, incluyendo enfermería, paramédicos, camilleros y choferes. Se consideraron diferentes turnos laborales: matutino, vespertino, nocturno y jornada acumulada. Se obtuvo información relacionada a la apreciación y frecuencia en que se presentó alguna lesión y dolor músculoesquelético en diferentes regiones anatómicas. También se evaluó

Tabla 2.

Frecuencia de dolor por región anatómica de los trabajadores según puesto de trabajo.

\begin{tabular}{|c|c|c|}
\hline \multicolumn{3}{|c|}{ Región Anatómica: Espalda Alta } \\
\hline Puesto & Frecuencia & Porcentaje \\
\hline Enfermeria & 1 & 9,09 \\
\hline Paramédico & 4 & 20,0 \\
\hline Camillero & 1 & 12,5 \\
\hline Chofer & 2 & 25,0 \\
\hline \multicolumn{3}{|c|}{ Región Anatómica: Espalda Baja } \\
\hline Enfermería & 2 & 18,1 \\
\hline Paramédico & 11 & 52,3 \\
\hline Camillero & 4 & 50,0 \\
\hline Chofer & 2 & 25,0 \\
\hline \multicolumn{3}{|c|}{ Región Anatómica: Cuello } \\
\hline Enfermería & 2 & 18,1 \\
\hline Paramédico & 4 & 19,0 \\
\hline Camillero & 0 & 0,0 \\
\hline Chofer & 1 & 12,5 \\
\hline \multicolumn{3}{|c|}{ Región Anatómica: Hombro } \\
\hline Enfermería & 0 & 0 \\
\hline Paramédico & 3 & 14,2 \\
\hline Camillero & 3 & 37,5 \\
\hline Chofer & 1 & 12,5 \\
\hline \multicolumn{3}{|c|}{ Región Anatómica: Brazos } \\
\hline Enfermería & 0 & 0 \\
\hline Paramédico & 0 & 0 \\
\hline Camillero & 1 & 12,5 \\
\hline Chofer & 0 & 0 \\
\hline \multicolumn{3}{|c|}{ Región Anatómica: Muñeca } \\
\hline Enfermería & 0 & 0 \\
\hline Paramédico & 0 & 0 \\
\hline Camillero & 1 & 12,5 \\
\hline Chofer & 0 & 0 \\
\hline \multicolumn{3}{|c|}{ Región Anatómica: Piernas } \\
\hline Enfermería & 4 & 36,3 \\
\hline Paramédico & 1 & 4,75 \\
\hline Camillero & 3 & 37,5 \\
\hline Chofer & 3 & 37,5 \\
\hline \multicolumn{3}{|c|}{ Región Anatómica: Pies } \\
\hline Enfermeria & 3 & 27,2 \\
\hline Paramédico & 3 & 14,2 \\
\hline Camillero & 4 & 50,0 \\
\hline Chofer & 1 & 12,5 \\
\hline
\end{tabular}

Los porcentajes calculados considerando el total de caso: 11 Enfermería, 21 Paramédicos, 8 Camilleros, 8 Choferes de Ambulancia y Traslado de Pacientes. 


\section{Gráfica 1.}

Frecuencia del dolor de espalda alta y antigüedad.

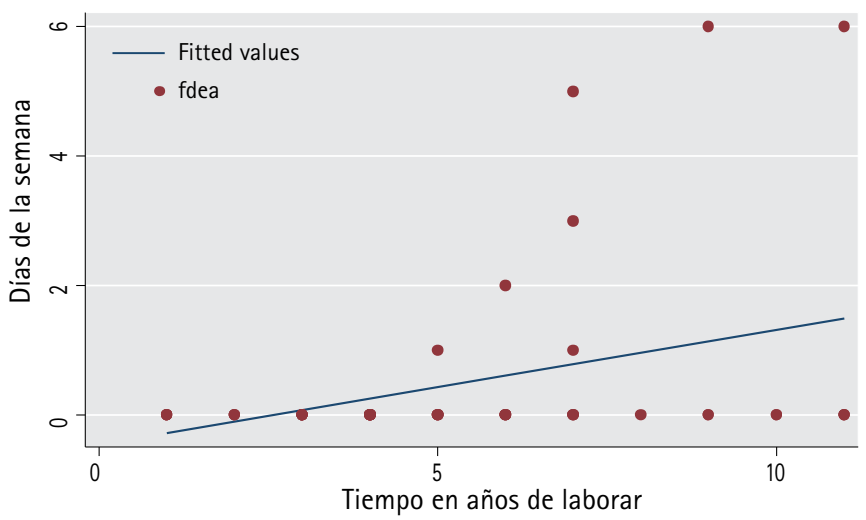

Gráfica 2.

Frecuencia del dolor de espalda baja y antigüedad.

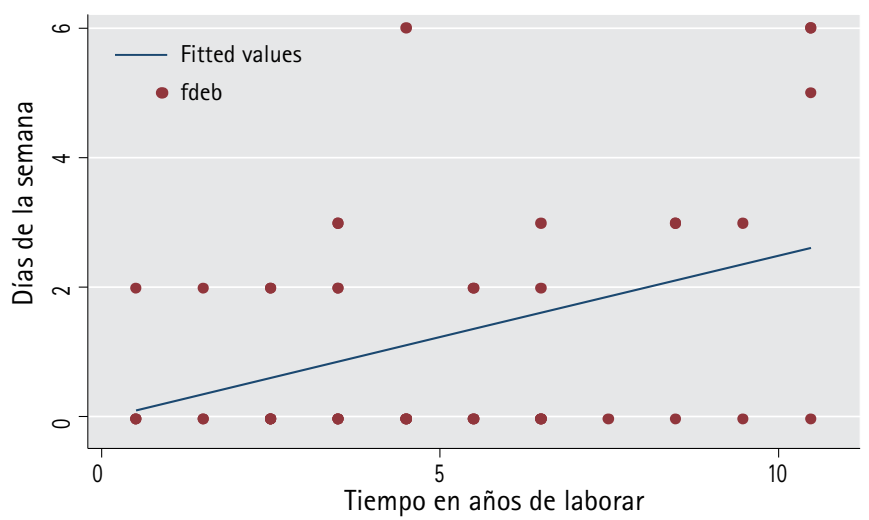

la información relacionada con la manipulación durante las cargas, capacitación y entrenamiento previo, el uso de equipo de protección personal para minimizar el riesgo de lesión, además de una sección de antecedentes personales no patológicos y patológicos. Como control de calidad del instrumento aplicado, se realizó una prueba piloto arrojando un valor alfa de Cronbach de 0,93 , considerando un resultado confiable para su aplicación por ser un valor mayor al $0,80 .{ }^{12}$

El análisis estadístico comprendió análisis descriptivo de las variables coeficientes de correlación entre la frecuencia de días que presenta el dolor, antigüedad y puesto desempeñado. Se realizó un análisis estratificado por grupo de sexo, actividad y turno laboral con el paquete estadístico STATA $10 .{ }^{13}$

\section{RESULTADOS}

Fueron 48 trabajadores los participantes incluidos, $29(60,42 \%)$ fueron hombres y $19(36,58 \%)$ mujeres. La media de edad fue de $29,1+6,7$ y $26,5+6,9$ respectivamente.

La Tabla 1 muestra la media y desviación estándar del peso y talla para ambos sexos. La edad se categorizó por rangos, el 47,91\% corresponde al grupo de 19 - 25 años. El turno con mayor actividad laboral fue el matutino (39,58\%), cuyas actividades incluyen cargar, trasladar y movilizar pacientes. El 43,75\% de la muestra fueron paramédicos seguido por enfermeria $(22,92 \%)$. Se categorizó el tiempo de
Gráfica 3.

Frecuencia del dolor en cuello y antigüedad.

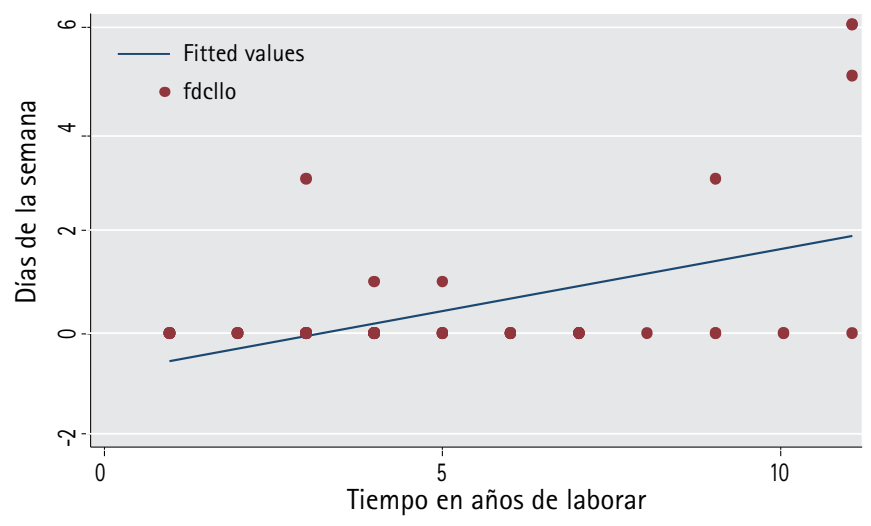

Gráfica 4.

Frecuencia del dolor en hombro y antigüedad.

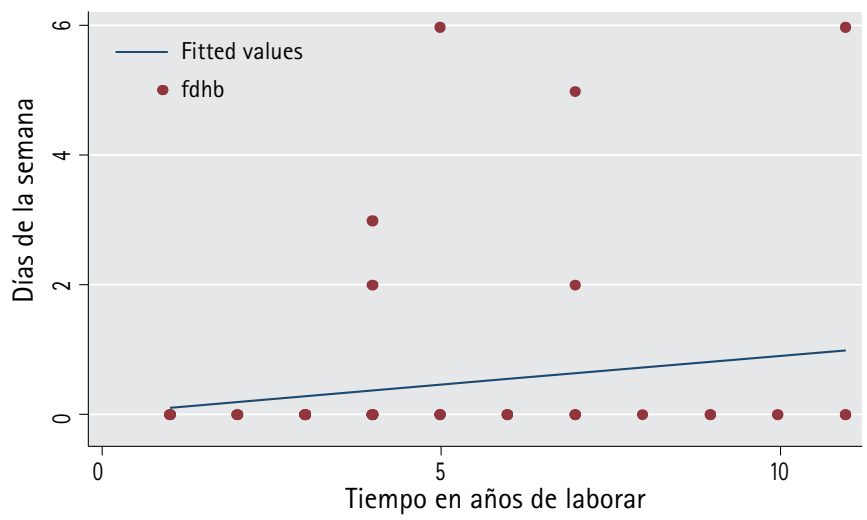

antigüedad dentro de la institución en años, siendo la más frecuente entre 1 - 5 años (56.25\%), y de 6 - 10 años (34,42\%).

La Tabla 2 muestra la frecuencia de dolor por región anatómica que presentaron los trabajadores según su actividad profesional. Los paramédicos presentaron la mayor frecuencia de dolor en espalda alta, seguida por espalda baja, cuello, hombros y pies.

Los trabajadores que realizan actividades como camilleros presentaron en segundo lugar la mayor frecuencia de dolor en espalda alta, seguido por espalda baja, hombro, brazo, muñeca, piernas y pies.

El personal de enfermería presentó menor manifestación de dolor comparada con las dos actividades anteriores, pero el dolor en piernas, pies, cuello y espalda baja se presentó con mayor frecuencia. Los choferes que trasladan pacientes, refirieron una menor proporción de dolor en piernas, espalda alta y espalda baja. La Gráfica 1 muestra la frecuencia de días de la semana que se presenta el dolor de espalda alta, lo cual se correlacionó con la antigüedad dentro de la institución. Se observó un incremento directamente proporcional en los días con dolor a los años de servicio. El mismo efecto se observa en las gráficas 2, 3 y 4 correspondiente al dolor en espalda baja, cuello y hombros respectivamente.

Al parecer no se encontró efecto para la frecuencia de dolor en brazo. Solo un caso presentó relación entre frecuencia del dolor y antigüedad (Gráfica 5). Lo anterior se observa también para el dolor en muñeca (Grafica 6).

Las gráficas 7 y 8 muestran un efecto negativo entre los días en que se presenta el dolor en piernas y pies contra la antigüedad. 


\section{Gráfica 5.}

Frecuencia del dolor en brazos y antigüedad.

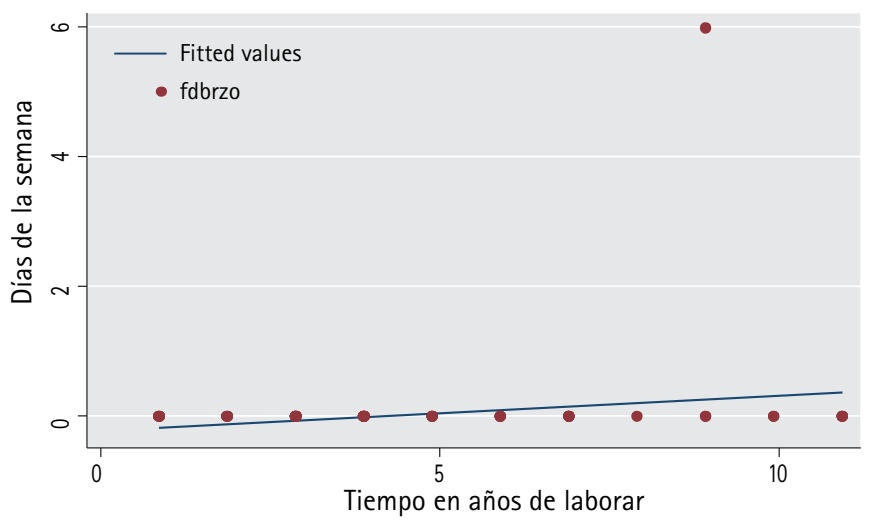

Gráfica 6.

Frecuencia del dolor en muñecas y antigüedad.

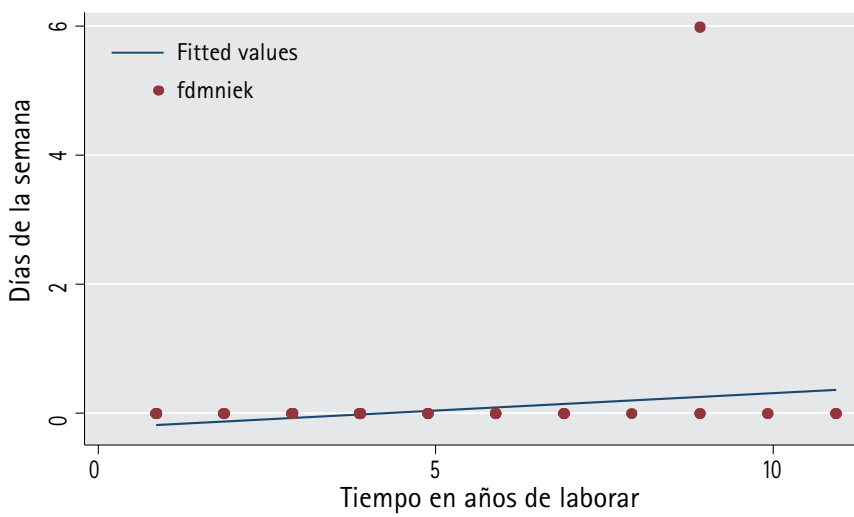

Tabla 3.

Correlaciones entre dolor en diferentes áreas anatómicas, el tiempo de laborar en la institución y el horario en que laboran.

$\begin{array}{lcc}\begin{array}{l}\text { Zona anatómica } \\ \text { donde se presenta el dolor }\end{array} & \begin{array}{c}\text { Tiempo de laborar } \\ \text { en la institución } \\ \% \text { de Correlación }\end{array} & \begin{array}{c}\text { Horario en que } \\ \text { labora el trabajador } \\ \% \text { de Correlación }\end{array} \\ \text { Espalda Alta } & 48,9^{* *} & 62,8^{¥ *} \\ \text { Espalda Baja } & 61,3^{* *} & 78,05^{\mp * *} \\ \text { Cuello } & 33,5^{*} & 8,27 \\ \text { Hombro } & 10,7^{*} & 4,11 \\ \text { Brazo } & 18,2 & 18,8^{\# *} \\ \text { Muñeca } & 21,5^{*} & 18,5^{¥ *} \\ \text { Piernas } & 7,9 & 5,04 \\ \text { Pies } & 66,0^{*} & 67,2^{\mp * *}\end{array}$

${ }^{*} p<0.01,{ }^{* *} p<0.001$

$¥$ Las correlaciones encontradas corresponden a los trabajadores del turno vespertino.

F Las correlaciones encontradas corresponden a los trabajadores del turno matutino.

El tiempo de laborar en la institución corresponde a un tiempo mayor de 10 años.

Tabla 4.

Correlación entre la frecuencia de dolor en dos regiones anatómicas que se presentan por semana en los trabajadores.

$\begin{array}{lccc} & \begin{array}{c}\text { Dolor de espalda alta } \\ \text { Dolor de hombro }\end{array} & \text { Dolor de brazo } & \text { Dolor de cuello } \\ & \begin{array}{r}62,02 \% \\ p=0,02\end{array} & \\ \text { Dolor de muñeca } & & 93,40 \% & \\ & & & \\ & & \\ \text { Dolor de pies } & & \begin{array}{l}67,000 \\ p=0,05\end{array}\end{array}$

Gráfica 7.

Frecuencia del dolor en piernas y antigüedad.

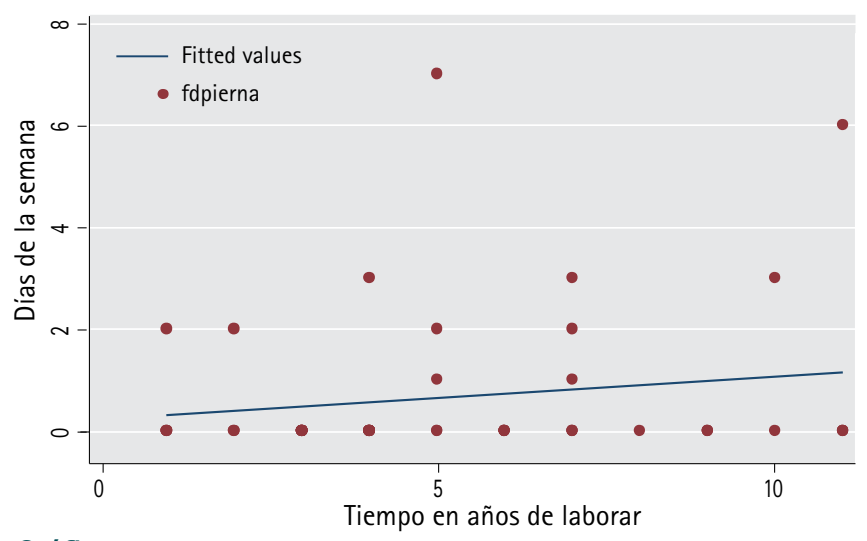

Gráfica 8.

Frecuencia del dolor en pies y antigüedad.

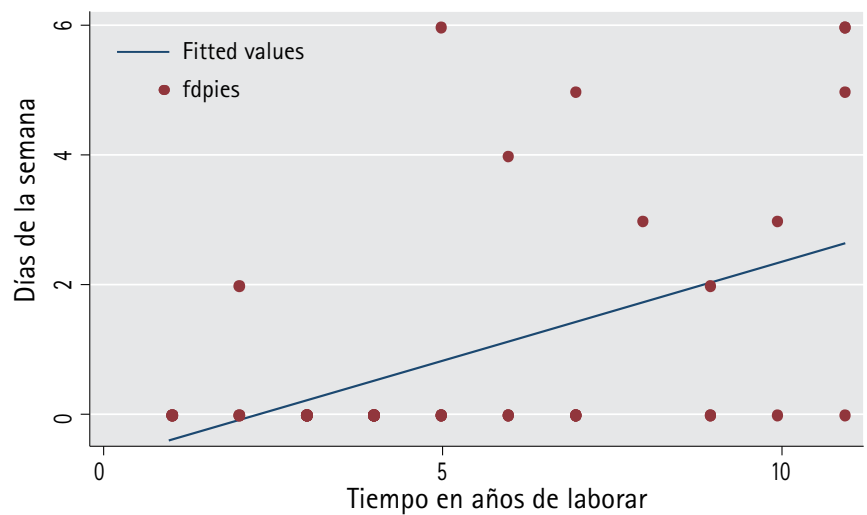

Se presentaron correlaciones significativas entre la antigüedad de los trabajadores y el incremento en días en que se presentó el dolor en espalda baja y alta de 48,9\% y $61,3 \%(\mathrm{p}<0,001)$ respectivamente (Tabla 3). Se observaron las correlaciones entre turno $u$ horario y la frecuencia de dolor en alguna región anatómica, encontrándose las más significativas eran los dolores en espalda alta y baja. Asímismo se observa la correlación con las variables antigüedad y horario de trabajo para dolor en pies.

La Tabla 4 muestra la correlación entre el dolor en diferentes localizaciones, pudiendo estar asociadas al dolor crónico. El dolor en brazos con dolor de muñeca mostraron una correlación del 93,4\% $(\mathrm{p}=0,000)$, seguido por el dolor en pies y cuello con una correlación de 67,6\% $\mathrm{p}=0,05$, por último una correlación del 62,0\% $(p=0,02)$ entre dolor de espalda alta y hombro.

El 95\% de los encuestados refirieron tener una capacitación o instrucción al ingreso, y solo un 7\% mencionó haber recibido cursos de seguridad e higiene, manejo de pacientes o higiene ocupacional por parte del hospital. Un 12\% refirió utilizar algún equipo de protección personal para realizar cargas, utilizadas por decisión personal y no por sugerencia del hospital ya que este no les proporciona este tipo de equipos.

\section{DISCUSIÓN}

La mayor parte de las enfermedades músculo-esqueléticas produce molestias o dolor local y en algunos casos hasta restricción de la 
movilidad, pudiendo obstaculizar el rendimiento normal en el trabajo o tareas de la vida diaria. Casi todos los padecimientos músculo-esqueléticos guardan relación con el trabajo, por lo que la actividad física puede agravarlas o provocar síntomas; sin embargo en la mayoría de los casos es imposible señalar un único factor causal. ${ }^{14}$ La mayoría de la población estuvo conformada por hombres y población en edad productiva. Esto puede ser determinante para lesiones a largo plazo si no se adoptan las medidas adecuadas. El turno matutino albergó la mayor proporción de trabajadores; pudiendo ser otra de las causas para la aparición de lesiones músculo-esqueléticas por ser un periodo de mayor actividad. Al analizar la distribución por área de servicio, los paramédicos tuvieron mayor proporción; seguido por el personal de enfermería, lo que puede favorecer a que se presenten más lesiones o dolores músculo-esqueléticos. Se encontró que el grueso de la población cuenta con un rango de uno a cinco años de antigüedad, lo cual se puede considerar como un periodo corto de exposición a las actividades laborales para la aparición prematura de dolores o lesiones músculo-esqueléticos ya que el periodo productivo laboralmente hablando en ambos sexos es de mínimo 28 años.

La media de la talla y el peso indica sobrepeso en los trabajadores, algunos de ellos con obesidad leve, por lo que puede interpretarse que dichas variables pudieran representar un indicador de riesgo para los dolores en espalda baja o lumbar. Duggleby y $\operatorname{Kumar}^{15}$ y Martínez PM. et al. ${ }^{14}$ reportan estos mismos hallazgos. Morata M. et al. ${ }^{16}$, Feuerstein M, et al. ${ }^{17}$ y Martínez PM. et al. ${ }^{14}$, reportaron que el personal paramédico y de enfermería presentan dolor con mayor frecuencia en espalda alta y baja, seguidos por cuello y hombros. Estos resultados son similares a los nuestros.

Dentro del personal de enfermería los dolores que con mayor frecuencia refirieron fue el dolor de piernas, pies y cuello, pudiendo estar relacionado con las actividades que realizan, sobre todo por los traslados intrahospitalarios, además de que se encuentran más tiempo de pie y el trabajo que desarrollan es en una sola postura. Montoya Díaz et al. ${ }^{18}$ y Valecillo M. et al. ${ }^{19}$ reportan hallazgos similares en los estudios realizados para evaluar lesiones osteomusculares en trabajadores de un hospital mexicano y venezolano respectivamente. Además encontraron que los trabajadores refirieron haber continuado con el dolor en los 12 meses posteriores a la primera manifestación. Nosotros encontramos resultados semejantes a los mencionados, pero al estratificar los días de la semana en que se presenta el dolor y a la antigüedad de los trabajadores en el hospital, se presentó un incremento en los días de la semana en que se manifiesta el dolor a medida que se incrementan los años de antigüedad. También encontramos correlaciones positivas para la aparición del dolor cuando estratificamos por turno laboral, lo cual puede ser una variable de importancia al tomar acciones preventivas dentro del personal hospitalario que realiza cargas, ya que es importante considerar que los turnos matutino y vespertino es cuando existe mayor actividad de atención a pacientes. Nuestros hallazgos se ven reforzados con lo reportado por De Souza et al. ${ }^{20}$ y Del Valle et al. ${ }^{21}$ quienes encontraron que los trabajadores de diferentes categorias profesionales y diversas actividades refirieron la permanencia de la sintomatología y la permanencia del dolor en días de la semana a medida que incrementan los años laborales. Hay que consideran que esto puede ser un reflejo de la falta de capacitación en relación a las cargas, situaciones ergonómicas y posturas corporales inadecuadas adoptadas durante los años trabajados. Aunque la mayoría de los trabajadores refieren haber tenido una capacitación previa para las actividades que realizarian, no se les capacitó en relación al desempeño de actividades de cargas pesadas ni se les proporciona una capacitación permanente sobre el uso de equipo de protección personal para la movilidad de pacientes o cargas. Aunque nuestro estudio cuenta con una muestra limitada, consideramos que abarcamos en gran medida la apreciación y lesiones que presentan los trabajadores. Es importante señalar el poco interés de los directivos hospitalarios para la participación de dicha investigación, por lo que es recomendable adoptar medidas de colaboración en pro de los trabajadores para evitar lesiones a largo plazo.

\section{CONCLUSIONES}

Los resultados obtenidos sugieren que el personal médico que realiza actividades diversas dentro del hospital sufre lesiones músculo-esqueléticas relacionadas con sus actividades laborales. La aplicación de intervenciones ergonómicas dentro del hospital puede resultar en cambios en el contenido de las actividades de trabajo, la adecuación del entorno laboral, la promoción de las condiciones de bienestar, seguridad y mantenimiento de la salud de los trabajadores, principalmente en el personal paramédico y de enfermería. Es importante considerar la utilización de herramientas auxiliares, por ejemplo en la movilidad y transferencia del paciente a su cama en forma manual requiere demasiado esfuerzo físico, sin embargo la utilización de ascensores hidráulicos requiere menor esfuerzo.

Por lo tanto, existe evidencia de la necesidad de correcciones de las intervenciones ergonómicas en el entorno del trabajo destinadas a mejorar las condiciones existentes y las intervenciones ergonómicas para la instrucción de los trabajadores sobre los riesgos a los que están expuestos y las formas de garantizar su labor sin lesiones. Estas medidas podrían prevenir el daño a largo plazo, minimizando el ausentismo laboral por causa de recuperación cuando existe lesión o cansancio; por consiguiente, manteniendo la productividad en el trabajo y ofrecer una mejor atención a los pacientes. 
1. Macatamney L, Corlett E. Ergonomic workplace assessment in a health care context. Ergonomics. 2000;35(9):965-8.

2. Verschuren $R$, Groot $B$, Nossent $S$. Working conditions in hospitals in the European Union. Dublin: European Foundation for the improvement of living and working conditions.1995.p. 93-113.

3. Hignett $S$, Macatamney L. Rapid entire body assessment (REBA). Appl Ergon. 2000;31:201-5.

4. Aranda J, Josa R, Nogareda S, Araujo C, Riba A. Lesiones de espalda y condiciones de trabajo en hospitales: Resultados. Salud y Trabajo. 1991; 83:22-6.

5. Garcia-Molina C, Chirivella C, Page A, Moraga R, Jorquera J. Evaluación de riesgos laborales asociados a la carga física. Paterna: Instituto de Biomecánica de Valencia (IBV); 1997.p. 111-22.

6. Nishide $V$, Benatti M. Riscos ocupacionais entre trabalhadores de enfermagem de uma unidade de terapia intensiva. Rev Esc Enferm USP. 2004; 38(4):406-14.

7. Botha $W$, Bridger R. Anthropometric variability, equipment usuability and musculoskeletal pain in a group of nurses in the Western Cape. Appl Ergon. 1998; 29:481-90.

8. Liga Reumatológica Española. Fisioterapia de patologias osteomusculares. Madrid: LIRE; 1983.

9. Miranda LR, Montero VM, Moya FN, Cruz MO. Incidencia de accidentes laborales en el personal de enfermeria del hospital Dr. Carlos Luis Valverde Vega, junio-agosto, San Ramón, 2005. Enfermería en Costa Rica. 2007; 28 (2):6-11.

10. Maldonado $A D$, Rodríguez $C A$. Causas básicas e inmediatas de riesgo de trabajo en el personal de enfermería. Hospital General de Zona. Congreso Nacional de Investigación IMSS; 1998; Tepic, Nayarit, México.

11. Kuorinka I, Jonsson B, Kilbom A, Vinterberg H, Biering-Sorensen F, Anderson G. et al. Standardised Nordic Questionaires for Analysis of Musculoskeletal Symptoms. Appl. Ergon. 1987;18(1):233-37.

12. Oviedo HC, Campos AA. Aproximación al uso del coeficiente alfa de Cronbach. Rev colomb psiquiatr. 2005; 34(5):572-80.
13. Stata Corp. Statisticas software; release 10.0. College Station: Stata Corporation; 2010.

14. Martínez Pérez MN, Vázquez Salvado M. Estudio epidemiológico del absentismo laboral en el personal hospitalario por dolor de espalda. Rev Rehabilitación. 2002; 36(3):137-42.

15. Duggleby T, Kumar S. Epidemiology of juvenile low back pain: a review. Disabil Rehabil. 1997 Dec;19(12):505-12.

16. Morata MA, Ferrer VA. Interacción entre estrés ocupacional, estrés psicológico y dolor lumbar: un estudio de profesionales sanitarios de traumatología y cuidados intensivos. MAPFRE Medicina. 2004;15(3):199-211.

17. Feuerstein $M$, Shaw WS, Nochola RA, Huang GD. From confounders to suspected risk factors: psychosocial factors and work-related upper extremity disorders J Electromyogr Kinesiol. 2004;14(1):171-178.

18. Montoya Díaz M, Palucci Marziale $M$, Do Carmo Cruz M, Taubert de Freitas F. Lesiones osteomusculares en trabajadores de un hospital Mexicano y la ocurrencia del ausentismo. Cienc Enferm. 2010;16(2):35-46.

19. Valecillo M, Quevedo A, Lubo A, Dos Santos A, Montiel M, Camejo M, et al. Síntomas musculo esqueléticos y estrés laboral en el personal de enfermería de un hospital militar. Salud de los Trabajadores. 2009;17(2):85-95.

20. De Souza C, Lima J, Antunez E, Schumacher K, Moreira R, De Almeida N. Riesgos ergonómicos de lesión por esfuerzo repetitivo del personal de enfermeria en el hospital. Enferm Global. 2011;23:251-62.

21. Royas A, Marziale M. A situacao de trabalho do pessoal de enfermegem no contexto de un hospital argentino: um estudio sob a otica da ergonomía. Rev Latino-am. Enfermagen. 2001;9(1):102-08.

\section{Referencia complementaria:}

Sluchak TJ. Ergonomics: Origins, focus and implementation considerations. AAOHNJ. 1992;40(3):105-112. 